\title{
Some consequences of the rank normal form of a matrix
}

\author{
Sorin Rădulescu \\ Institute of Mathematical Statistics \\ and Applied Mathematics, \\ Bucharest, Romania \\ email: xsradulescu@gmail.com
}

\author{
Marius Drăgan \\ Department of Mathematics, \\ "Mircea cel Bătrân" Technical College, \\ Bucharest, Romania \\ email: dragan2005@yahoo.com
}

\author{
Mihály Bencze \\ Áprily Lajos High school, Braşov, Romania \\ email: 1benczemihaly@gmail.com; \\ benczemihaly@yahoo.com
}

\begin{abstract}
If $\mathrm{A}$ is a rectangular matrix of rank $\mathrm{r}$, then $\mathrm{A}$ may be written as PSQ where $P$ and $Q$ are invertible matrices and $S=\left(\begin{array}{ll}I_{r} & O \\ O & O\end{array}\right)$. This is the rank normal form of the matrix $\mathrm{A}$.

The purpose of this paper is to exhibit some consequences of this representation form.
\end{abstract}

\section{Introduction and notation}

In the following we shall denote by $K$ a commutative field and by $M(m \times n, K)$ the set of matrices with $m$ lines and $n$ columns with elements in $K$. By $\mathrm{O}_{m, n}$ we shall denote the null matrix with $\mathrm{m}$ lines and $\mathrm{n}$ columns and by $\mathrm{I}_{\mathrm{r}, \mathrm{r}}$ the unit matrix with $\mathrm{r}$ lines and $\mathrm{r}$ columns.

The purpose of this paper is to exhibit some consequences of the following representation theorem from [1], [2], [3], [4], [5], [6], [7]. 
Theorem 1 (rank normal form of a matrix). Let $A \in M(m \times n, K)$ with rankA $=r$. Then there are the invertible matrices $\mathrm{P} \in \mathrm{M}(\mathrm{m} \times \mathrm{m}, \mathrm{K})$ and $\mathrm{Q} \in \mathrm{M}(\mathrm{n} \times \mathrm{n}, \mathrm{K})$ and

$$
S=\left[\begin{array}{ll}
\mathrm{I}_{r, r} & \mathrm{O}_{r, n-r} \\
\mathrm{O}_{\mathrm{m}-r, r} & \mathrm{O}_{\mathrm{m}-\mathrm{r}, \mathrm{n}-\mathrm{r}}
\end{array}\right] \in M(\mathrm{~m} \times \mathrm{n}, \mathrm{K})
$$

such that $\mathrm{A}=\mathrm{PSQ}$.

The exposition of the results is made in an unitary manner using the block matrices and contains some known inequalities as inequality of Sylvester and inequality of Frobenius.

The Theorem 9 is new.

\section{Main results}

In the following we give three representation theorems 2,3 and 4 which are direct consequences of Theorem 1 .

Theorem 2 Let $A \in M(m \times n, K)$ with $\operatorname{rank} A=r$. Then there are $A_{1}, A_{2}, \ldots$, $A_{r} \in M(m \times n, K)$ matrices of rank 1 such that $A=A_{1}+A_{2}+\ldots+A_{r}$.

Proof. The proof follows from Theorem 1 if we note that the matrix

$$
S=\left[\begin{array}{ll}
I_{r, r} & O_{r, n-r} \\
O_{m-r, r} & O_{m-r, n-r}
\end{array}\right]
$$

may be written as the sum of $r$ matrices of rank 1 .

Theorem 3 Let $A \in M(m \times n, K)$ with $\operatorname{rank} A=r$. Then there are two matrices with rank equal with $\mathrm{r}, \mathrm{B} \in \mathrm{M}(\mathrm{m} \times \mathrm{r}, \mathrm{K})$ and $\mathrm{C} \in \mathrm{M}(\mathrm{r} \times \mathrm{n}, \mathrm{K})$ such that $\mathrm{A}=\mathrm{BC}$.

Proof. From Theorem 1 it follows that there are the invertible matrices $\mathrm{P} \in$ $M(m \times m, K)$ and $Q \in M(n \times n, K)$ and

$$
S=\left[\begin{array}{ll}
\mathrm{I}_{r, r} & \mathrm{O}_{r, n-r} \\
\mathrm{O}_{\mathrm{m}-\mathrm{r}, \mathrm{r}} & \mathrm{O}_{\mathrm{m}-\mathrm{r}, \mathrm{n}-\mathrm{r}}
\end{array}\right] \in \mathrm{M}(\mathrm{m} \times \mathrm{n}, \mathrm{K})
$$

such that $A=P S Q$. 
Note that $S=S_{1} S_{2}$ where $S_{1}=\left[\begin{array}{l}I_{r, r} \\ O_{m-r, r}\end{array}\right] \in M(m \times r, K)$ and $S_{2}=$ $\left[\mathrm{I}_{r, r}, \mathrm{O}_{\mathrm{r}, \mathrm{n}-\mathrm{r}}\right] \in \mathrm{M}(\mathrm{r} \times \mathrm{n}, \mathrm{K})$.

If we put $B=P S_{1}$ and $C=S_{2} Q$ we have $A=B C$ and $\operatorname{rank} B=\operatorname{rank}\left(P_{1}\right)=$ $\operatorname{rank} S_{1}=r, \operatorname{rank} C=\operatorname{rank}\left(S_{2} Q\right)=\operatorname{rank} S_{2}=r$.

Theorem 4 Let $A \in M(n \times n, K)$ with rank $A=r$. Then there exists two matrices with rank $\mathrm{r}, \mathrm{B} \in \mathrm{M}(\mathrm{n} \times \mathrm{n}, \mathrm{K})$ and $\mathrm{C} \in \mathrm{M}(\mathrm{n} \times \mathrm{n}, \mathrm{K})$ such that $\mathrm{A}=$ BC.

Proof. From Theorem 1 it follows that there are two invertible matrices $\mathrm{P}, \mathrm{Q} \in$ $M(n \times n, K)$ and $S=\operatorname{diag}\left[I_{r, r}, O_{n-r, n-r}\right]$ such that $A=P S Q$. Let $B=P S$ and $\mathrm{C}=\mathrm{SQ}$.

From the equality $S^{2}=S$ we have that $A=B \cdot C$. We have

$\operatorname{rank} B=\operatorname{rank}(P S)=\operatorname{rank} S=r$ and $\operatorname{rank} C=\operatorname{rank}(S Q)=\operatorname{rank} S=r$

Lemma 1 Let $A \in M(m \times n, K), B \in M(p \times q, K), S=\left[\begin{array}{ll}I_{r, r} & O_{r, p-r} \\ O_{n-r, r} & O_{n-r, p-r}\end{array}\right]$ $\in M(n \times p, K), S_{1}=\left[\begin{array}{l}I_{r, r} \\ O_{n-r, r}\end{array}\right] \in M(n \times r, K), S_{2}=\left[I_{r, p}, O_{r, p}\right] \in M(r \times p, K)$.

Then the following statements are true:

i). $\operatorname{rank}(A S) \geq \operatorname{rank} A+r-n$

ii). $\operatorname{rank}\left(A S_{1}\right)=\operatorname{rank}\left(A S_{1} S_{2}\right)$

iii). $\operatorname{rank}\left(S_{1} S_{2} B\right)=\operatorname{rank}\left(S_{2} B\right)$.

\section{Proof.}

i). Let $A=\left[\begin{array}{ll}A_{1} & A_{2} \\ A_{3} & A_{4}\end{array}\right]$ where $A_{1} \in M(r \times r, K), A_{2} \in M(r \times(n-r), K)$, $A_{3} \in M((m-r) \times r, K), A_{4} \in M((m-r) \times(n-r), K)$.

We have

$$
\begin{aligned}
\operatorname{rank}(A S) & =\operatorname{rank}\left(\left[\begin{array}{ll}
A_{1} & A_{2} \\
A_{3} & A_{4}
\end{array}\right] \cdot\left[\begin{array}{ll}
I_{r, r} & O_{r, p-r} \\
O_{n-r, r} & O_{n-r, p-r}
\end{array}\right]\right) \\
& =\operatorname{rank}\left[\begin{array}{ll}
A_{1} & O_{r, p-r} \\
A_{3} & O_{n-r, p-r}
\end{array}\right]=\operatorname{rank}\left(\left[\begin{array}{l}
A_{1} A_{2} \\
A_{3} A_{4}
\end{array}\right]-\operatorname{rank}\left[\begin{array}{ll}
O_{r, r} & A_{2} \\
O_{m-r, r} & A_{4}
\end{array}\right]\right)
\end{aligned}
$$




$$
\geq \operatorname{rank}\left[\begin{array}{ll}
A_{1} & A_{2} \\
A_{3} & A_{4}
\end{array}\right]-\operatorname{rank}\left[\begin{array}{l}
A_{2} \\
A_{4}
\end{array}\right] \geq \operatorname{rank} A+r-n
$$

ii). We have

$$
\begin{aligned}
\operatorname{rank}\left(A S_{1}\right) & =\operatorname{rank}\left(\left[\begin{array}{ll}
A_{1} & A_{2} \\
A_{3} & A_{4}
\end{array}\right]\left[\begin{array}{l}
I_{r, r} \\
O_{n-r, r}
\end{array}\right]\right)=\operatorname{rank}\left[\begin{array}{l}
A_{1} \\
A_{3}
\end{array}\right] \\
& =\operatorname{rank}\left(\left[\begin{array}{ll}
A_{1} & A_{2} \\
A_{3} & A_{4}
\end{array}\right]\left[\begin{array}{ll}
I_{r, r} & O_{r, p-r} \\
O_{n-r, r} & O_{n-r, p-r}
\end{array}\right]\right)=\operatorname{rank}\left(A S_{1} S_{2}\right)
\end{aligned}
$$

iii). We can write

$$
\mathrm{B}=\left[\begin{array}{ll}
\mathrm{B}_{1} & \mathrm{~B}_{2} \\
\mathrm{~B}_{3} & \mathrm{~B}_{4}
\end{array}\right]
$$

where $B_{1} \in M(r \times r, K), B_{2} \in M(r \times q-r, K), B_{3} \in M((p-r) \times r, K)$, $B_{4} \in M((p-r) \times q-r, K)$.

We have

$$
\begin{aligned}
\operatorname{rank}\left(S_{1} S_{2} B\right) & =\operatorname{rank}(S, B)=\operatorname{rank}\left(\left[\begin{array}{ll}
I_{r, r} & O_{r, p-r} \\
O_{n-r, r} & O_{n-r, p-r}
\end{array}\right]\left[\begin{array}{ll}
B_{1} & B_{2} \\
B_{3} & B_{4}
\end{array}\right]\right) \\
& =\operatorname{rank}\left[\begin{array}{ll}
B_{1} & B_{2} \\
O_{p-r, r} & O_{p-r, q-r}
\end{array}\right]=\operatorname{rank}\left[B_{1} B_{2}\right] \\
& =\operatorname{rank}\left(\left[I_{r, r}, O_{r, p-r}\right]\left[\begin{array}{ll}
B_{1} & B_{2} \\
B_{3} & B_{4}
\end{array}\right]\right)=\operatorname{rank}\left(S_{2} B\right) .
\end{aligned}
$$

Theorems 5 and 6 may be found in the papers [1], [2], [3], [4], [5], [6], [7].

Theorem 5 (Sylvester). Let $\mathrm{A} \in \mathrm{M}(\mathrm{m} \times \mathrm{n}, \mathrm{K}), \mathrm{B} \in \mathrm{M}(\mathrm{n} \times \mathrm{p}, \mathrm{K})$. Then is true that

$$
\operatorname{rank}(A B) \geq \operatorname{rank} A+\operatorname{rank} B-n
$$

Proof. The rank normal form of matrix $B$ is $B=P S Q$, rank $B=r$ where $P \in M(n \times n, K)$ and $Q \in M(p \times p, K)$ are invertible and

$$
S=\left[\begin{array}{ll}
\mathrm{I}_{r, r} & \mathrm{O}_{r, p-r} \\
\mathrm{O}_{n-r, r} & \mathrm{O}_{n-r, p-r}
\end{array}\right] \in M(\mathrm{n} \times \mathrm{p}, \mathrm{K}) .
$$

According with Lemma 1 i). we have

$$
\begin{aligned}
\operatorname{rank}(A B) & =\operatorname{rank}(A P S Q)=\operatorname{rank}(A P S) \geq \operatorname{rank}(A P)+r-n \\
& =\operatorname{rank} A+\operatorname{rank} B-n
\end{aligned}
$$


Theorem 6 (Frobenius). Let $\mathrm{A} \in \mathrm{M}(\mathrm{m} \times \mathrm{n}, \mathrm{K}), \mathrm{B} \in \mathrm{M}(\mathrm{n} \times \mathrm{p}, \mathrm{K}), \mathrm{C} \in \mathrm{M}$ $(\mathrm{p} \times \mathrm{q}, \mathrm{K})$ then the following inequality is true

$$
\operatorname{rank}(A B)+\operatorname{rank}(B C) \leq \operatorname{rank} B+\operatorname{rank}(A B C)
$$

Proof. Let $r=\operatorname{rank} B$ and the rank normal form of $B$ is $B=$ PSQ where $P \in M(n \times n, K)$ and $Q \in M(p \times p, K)$ are invertible. We shall apply Lemma 1, ii). and iii). and Sylvester inequality and we will obtain:

$$
\begin{aligned}
\operatorname{rank}(A B C) & =\operatorname{rank}(A P S Q C)=\operatorname{rank}\left(A P S, S_{2} Q C\right) \\
& \geq \operatorname{rank}\left(A P S_{1}\right)+\operatorname{rank}\left(S_{2} Q C\right)-r \\
& =\operatorname{rank}\left(A P S_{1} S_{2}\right)+\operatorname{rank}\left(S_{1} S_{2} Q C\right)-r \\
& =\operatorname{rank}(A P S Q)+\operatorname{rank}(P S Q C)-r \\
& =\operatorname{rank}(A B)+\operatorname{rank}(B C)-\operatorname{rank} B
\end{aligned}
$$

Theorem 7 Let $\mathrm{A} \in \mathrm{M}(\mathrm{n} \times \mathrm{n}, \mathrm{K})$ and the sequence $\left(\mathrm{a}_{\mathrm{p}}\right)_{\mathrm{p} \geq 1}$ defined by $\mathrm{a}_{\mathrm{p}}=$ $\operatorname{rank}\left(A^{p}\right), p \geq 1$. Then the following statements hold:

i). $\left(a_{\mathfrak{p}}\right)_{\mathrm{p} \geq 1}$ is decreasing

ii). $2 a_{p+1} \leq a_{p}+a_{p+2}$ for each $p \geq 1$

iii). $a_{\mathfrak{p}}=a_{\mathfrak{p}+1}$ implies that $\mathrm{a}_{\mathfrak{p}}=\mathrm{a}_{\mathrm{p}+\mathrm{t}}$ for each $\mathrm{t} \geq 1$.

Proof. i). We have

$$
a_{\mathfrak{p}+1}=\operatorname{rank}\left(A^{p+1}\right) \leq \min \left(\operatorname{rank}(A)^{p} ; \operatorname{rank} A\right) \leq \operatorname{rank}\left(A^{p}\right)=a_{\mathfrak{p}}
$$

for each $p \geq 1$.

ii). From Theorem 5 we have for $p \geq 1$

$$
\operatorname{rank}\left(A A^{p}\right)+\operatorname{rank}\left(A^{p} A\right) \leq \operatorname{rank}\left(A^{p}\right)+\operatorname{rank}\left(A A^{p} A\right)
$$

this inequality is equivalent with

$$
2 a_{p+1} \leq a_{p}+a_{p+2}
$$

for each $p \geq 1$.

iii). It results from i) and ii). 
Theorem 8 Let $\mathrm{A} \in \mathrm{M}(\mathrm{n} \times \mathrm{n}, \mathrm{K})$ and $\mathrm{q}_{\mathrm{A}}$ the minimal polynomial of the matrix $A$. Then the following inequality hold

$$
\operatorname{deg}\left(q_{A}\right) \leq 1+\operatorname{rank} A
$$

Proof. We de note

$$
\mathrm{r}=\operatorname{rank} A
$$

From Theorem 2 it follows that it exists $B \in M(n \times r, K)$ and $C \in M(r \times n, K)$ such that $A=B C$.

Let $D=C B \in M(r \times r, K)$ and $f_{D}(t)=\operatorname{det}\left(t I_{r}-D\right), t \in K$.

We have from Hamilton-Cayley-Frobenius theorem that

$$
f_{D}(D)=O_{r}
$$

It follows that

$$
\mathrm{Bf}_{\mathrm{D}}(\mathrm{CB}) \mathrm{C}=\mathrm{O}_{\mathrm{n}}
$$

Because $B(C B)^{p} C=(B C)^{p} B C$ for $p \geq 1$.

We obtain that

$$
f_{D}(B C) B C=O_{n}
$$

Let $g(t)=t f_{D}(t), t \in K$ and note that $\operatorname{deg} g=r+1$. We have $g(A)=O_{n}$ and that $q_{A} \mid g$, so

$$
\operatorname{deg} q_{A} \leq \operatorname{deg} g=r+1=1+\operatorname{rank} A
$$

Theorem 9 Let $\mathrm{A} \in \mathrm{M}(\mathrm{n} \times \mathrm{n}, \mathrm{K})$. Then the following statement are equivalent

i). $A^{2}=O_{n}$

ii). There are $\mathrm{B}, \mathrm{C} \in \mathrm{M}(\mathrm{n} \times \mathrm{n}, \mathrm{K})$ with the following properties

$$
\mathrm{A}=\mathrm{BC} \text { and } \mathrm{CB}=\mathrm{O}_{\mathrm{n}}
$$

Proof. ii). $\Rightarrow$ i). Let $A=B C$ with $C B=O_{n}$. We have

$$
A^{2}=B C B C=B_{n} C=O_{n}
$$

i). $\Rightarrow$ ii). Let $A=$ PSQ the rank normal form of A. Note that $S^{2}=S$. We put $B=P S$ and $C=S Q$. We have

$$
\mathrm{O}_{\mathrm{n}}=\mathrm{A}^{2}=\mathrm{BCBC}=\mathrm{PSSQPSSQ}=\mathrm{PSQPSQ}
$$

Because $P$ and $Q$ are invertible, we obtain that $S Q P S=O_{n}$. It follows that $\mathrm{CB}=\mathrm{O}_{n}$ and $\mathrm{A}=\mathrm{BC}$. 


\section{References}

[1] J. E. Gentle, Matrix algebra theory, Computations and applications in statistics, 2nd Edition Springer (2007).

[2] R. A. Horn, C. R. Johnson, Matrix analysis, 2nd Edition, Camridge University Press (2013).

[3] P. Lancaster, T. Tismentesky, The theory of matrices, 2nd Edition, Computer science and applied mathematics (1985).

[4] C. D Meyer, Matrix analysis and applied linear algebra, SJAM (2010).

[5] D. Serre, Matrices theory and applications, G.T.M. Springer (2016).

[6] R. S. Shayle, A. Khury, Matrix algebra useful for statistics, J. Wiley (2017).

[7] F. Zhang, Matrix theory, Basic results and techniques, Springer (1999). 